\title{
Researching Algorithms and Recommendation Systems Inside Out Using Reversed Engineering
}

\author{
Lene Pettersen \\ Department of Informatics, University of Oslo, Norway \\ Lenpe@ifi.uio.no
}

\begin{abstract}
It is difficult for a lone researcher to obtain an unfiltered perspective of algorithms in digital media and recommendation systems in digital platforms because these companies tend to be reluctant to share insights into their algorithms and business models. Researchers therefore need to develop new methods to obtain knowledge. The method of reversed engineering, which explores an algorithm from the inside out by "re-engineering" how algorithms are set up, has been recommended for gaining empirical knowledge about how algorithms work and what they do. This paper uses reversed engineering to illustrate how algorithms in dating platforms calculate matches between single persons. One of the findings is that the matching algorithms in dating platforms follows a psychological discourse based on similarities in personality and other personal aspects between candidates when recommending matches, and thus ignoring socioeconomic principles (e.g. economic income, social class, educational level) that social science find important when choosing a life-long partnership. Another observation is that an algorithmic matching machinery based on similarities follows a 'more of the same' logic, which risks limiting the pool of single candidates the user gain access to.
\end{abstract}

\section{Introduction}

Many of our daily activities and much of our media consumption are increasingly being shaped by algorithms operating behind the scenes. Examples include the selection of online news via search engines and news aggregators, the consumption of music and video entertainment via recommendation systems, the choice of services and products in online shops, and the selection of status messages displayed on social online networks [1]. Algorithms recommend friends, news, songs, travel routes, and potential partners, and they calculate scorings of content and people presented in ranked lists [1]. With the rise of YouTube, Amazon,
Netflix, and many other Web services, recommendation systems have taken an increasing role in our lives. Recommendation systems are essentially algorithms that have been developed to suggest to the user relevant items, such as movies to watch, text to read, and products to buy [2].

Attention from a variety of disciplinary communities (e.g., information systems, strategy and digital economy, and media science) has in past years been directed towards algorithms and how they work. However, platform owners are often reluctant to share information on how their algorithms are set up [3] because this typically involves revealing their business models and secrets. Powerful platforms make the decisions which order public culture, but do not disclose what is beneath their decision-making processes [4]. Hence, the public never quite know how algorithmic decisions are being made, yet one can assume they serve the strategic commercial interests of the platforms [4]. In a general sense, 'algorithmic culture' denote the use of computational processes to sort, classify, and hierarchise people, places, objects, and ideas, as well as the habits of thought, conduct and expression that arise in relationship to those processes [5]. Thus, although an algorithm is commonly understood as a set of defined steps to produce particular outputs, producing algorithms requires a great deal of expertise and involves many judgements, choices, and constraints [6, 7]. As example, Facebook's algorithms weight content based on popularity and engagement (likes, comments etc.). Facebook thus constructs a regime of visibility which imposes a 'threat of invisibility.' As a result, there is a constant possibility that content shared disappears and becomes obsolete, risking that some content might never be shown in newsfeeds at all [8]. Similarly, Tinder uses an Elo score (from Chess rankings) as its matching algorithm, where people with a low ranking will only be able to see other people who also have a low ranking [9]. Thus, the algorithmic machinery of platforms such as Facebook or Tinder (as well as other platforms) control availability and accessibility to the pool of content or persons the userget access to. 
To gain a wider understanding of how algorithms in different digital media and platforms operate, more studies are needed to investigate how algorithms work and identify which intentions, values, and constraints they potentially hold [10,11]. However, it is difficult for a lone researcher to obtain an unfiltered perspective of algorithms in recommendation systems [3]. As a result, research undertaken around, for example, algorithmic music discovery has been done from a strict technical perspective within the field of computer science [12]. Because of the difficulty obtaining valid data from streaming services [3], researchers need to develop new methods to obtain insights into how algorithms work [10]. Reversed engineering has been suggested as a suitable method for gaining empirical knowledge about how algorithms work and what they do [10, 13]. Reversed engineering explores an algorithm from the inside out by "re-engineering" how algorithms are set up. Empirical work using this method is called for $[6,10]$ because it can inspire how we research social and digital media and platforms. This paper presents in detail how the method of reversed engineering was used to understand the algorithmic matching machinery in four online dating platforms. Only a few studies have used this method or shown empirically step-by-step how the reversed engineering method can be employed when researching social media and online platforms. One exception is Snickars [12] that used reverse engineering to study Spotify's aggregation procedures, metadata, valuation strategies, the hidden infrastructures of digital music distribution, and its underlying norms and structures. However, Spotify's recommender system uses a collaborative filtering method (recommendations are based on previous interactions and history), while algorithms in dating platforms use a content-based model (recommendations are based on the information the platform has in its database).

This paper presents in detail the reversed engineering method used in a study of four online dating platforms. By using this method, insights into how algorithms are set up to calculate and recommend matches in dating platforms were obtained. This study also reveals how algorithms are biased by certain theoretical perspectives and models when calculating matches between single persons. Before I show how the method of reversed engineering was conducted in practice, I present the key aspects of algorithms and recommendation systems and the domain knowledge on how people choose long-lasting relationships or partners from a social science and socioeconomic perspective.

\section{Algorithms and recommendation systems}

From e-commerce (which suggests to buyers' articles that could interest them) to online advertisement (which suggests to users the right contents based on previous preferences), recommendation systems are currently unavoidable in our daily online journeys [2]. Similarly, meeting a potential partner has been reshaped with the advent of big data and algorithmic matchmaking, and the dating industry and platforms is attracting an increasing number of customers [14]. By 2007, online dating had become the second highest online industry for paid content [15], and online dating platforms represent significant business opportunities, where love is only a few clicks and dollars away [16-18].

In simple terms, an algorithm is a well-defined computational procedure that takes some value or set of values as the input and produces some value or set of values as the output $[3,10]$. An algorithm is thus a sequence of computational steps that transforms the input into the output [3]. Algorithms-based on input from programmers and platform owners-sort, choose, and discriminate between different types of content and information [10].

Different types of algorithms exist. Just and Latzer [13] identified nine main types of algorithms: (1) search (e.g., general search engines), (2) aggregation (e.g., news aggregators), (3) observation/surveillance (e.g., employee monitoring), (4) prognosis/forecast (e.g., predictive policing), (5) filtering (e.g., spam filters), (6) recommendation (e.g., recommender systems), (7) scoring (e.g., reputation systems), (8) content production (e.g., algorithmic journalism), and (9) allocation (e.g., computational advertising).

The sixth type-recommendation systems [19]has proved in recent years to be a valuable means for coping with the information overload problem [20] and for suggesting relevant items or content, such as movies to watch, text to read, and products to buy [2]. Two types of recommender methods are used to suggest relevant items to users: (a) collaborative filtering methods and (b) content-based methods [2]. Collaborative methods are based solely on past interactions recorded between users and items (e.g., likes, browsing history, and watched movies) to produce new recommendations, whereas content-based approaches use additional information about users and/or items (e.g., age, gender, and preferences) to model their recommendations. Dating platforms (e.g., Match or eHarmony) typically use a content-based model when calculating good matches between single persons. Users are asked for information about themselves, their partner preferences and other 
personal information, such as age, height, education, and personality aspects, when creating their online profiles on dating platforms. However, due to selfpresentation concerns, users of datingplatforms could underreport socially undesirable activities and overreport socially desirable ones, what is known as the social desirability bias [21]. Interestingly, Joel, Eastwick and Finkel [22] used machine learning to test how well people's self-reported traits and preferences predict people's overall tendencies to romantically desire other people and to be desired by other people, amongst others. They created an algorithm to match participants based on their self-reported personality tastes. The system, however, could not predict who ended up pairing off [22].

As collaborative methods for recommendation systems base their recommendations completely on past interactions recorded between users and items [2], these methods suffer from the "cold start problem," which refers to the problem of recommending something to new users for whom a history on which to base the prediction is lacking [2]. Unlike collaborative methods that rely solely on user-item interactions, content-based approaches use additional information about the users and/or items [2]. In a movie recommender system, the additional information could be the user's age, gender, or occupation as well as the category, main actors, and duration of the movie (items) [2]. Content-based methods suffer less from the cold start problem than collaborative approaches because new users or items can be described by their characteristics (content) and relevant suggestions can thus be made based on these new entities. In contentbased methods, the recommendation problem is classified as either a classification problem (predict whether a user "likes" an item) or a regression problem (predict the rating given by a user for an item) [2].

\subsection{Reversed engineering}

Of several methodological approaches to gain a wider understanding of how algorithms in different digital media and platforms work, Bucher [6] suggested using a cybernetic trace and observing different feedback mechanisms between the systems and their surroundings. This method of reversing the data engineer's constructions is known as reversed engineering. This method allows us to direct our attention to what the algorithm does, rather than what it is, by making the "black box" available through observable leaks, openings, and shortcomings. By directing our attention to the systems' functions rather than their actual content, reversed engineering enables us to experiment with the inputs and outputs of the boxes and examine the outcome of the users' choices and interactions with the system. The overall goal is to critically and better understand algorithms' mechanisms. Reversed engineering, which explores an algorithm from the inside out, can thus help to obtain a better understanding of how algorithms empirically work [6, 10], which is critical given the increasing importance of algorithms in shaping social and economic life. Algorithms do not operate in a technical vacuum and cannot be divorced from the underlying perspectives upon which the system is modelled because the code, algorithms, and computer systems contain the embedded values held by the individuals who construct them [23-25].

Reversed engineering can also be described as a "process of articulating the specifications of a system through a rigorous examination drawing on domain knowledge, observation, and deduction to unearth a model of how that system works" [26]. Snickars [12] used reversed engineering to study Spotify. By creating 150 automated bots that interacted differently with music, he gained key insights into Spotify's recommendation algorithms.

The functioning of algorithmic selection can be described as a basic input-throughput-output model that forms the basis for reversed engineering (Figure 1) [13].

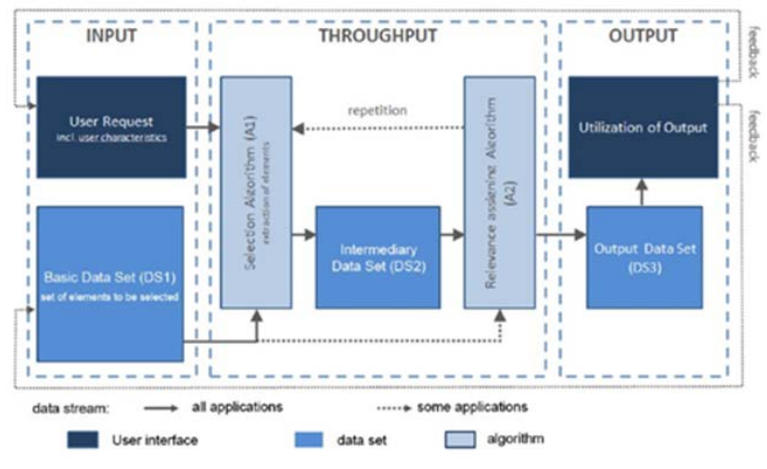

\section{Figure 1: Input-throughput-output model of algorithmic selection on the internet (adapted from Just and Latzer [13]).}

Algorithms form the centerpiece of the throughput stage where they operate. Starting from the available user characteristics, they apply statistical operations to select elements from a basic data set (DS1) and assign relevance to them. The throughput process is characterized by the assignment of relevance (A2) and respective selections (A1), based on a multitude of different codes and operating modes (e.g., matching or 
sorting algorithms). The output (DS3) also assumes different forms (e.g., rankings, recommendations) [13].

\subsection{Domain knowle dge}

Diakopoulos [26] highlighted the importance of having domain knowledge when doing reversed engineering: Reversed engineering is a "process of articulating the specifications of a system through a rigorous examination drawing on domain knowledge, observation, and deduction to unearth a model of how that system works” (p. 13). This study identifies how a platform's algorithms calculate matches and examines the extent to which dating platforms consider socioeconomic aspects the literature finds important when heterosexual people choose a partner for a longterm relationship. Two such aspects relate to the principles of homogamy and hypergamy [27]. Homogamy denotes marriage between individuals who are, in some way, similar to each other. Hypergamy is the sociological tendency that women choose partners of the same or higher social status as themselves, while men do the opposite [27].

Socioeconomic aspects of the hypergamy principle can be, with the lens of Bourdieu's forms of capital [28], observed and "measured" based on variables or capital, such as educational level, occupation, occupational level or rank (e.g., manager, blue-collar worker), and/or income. Height is a biological capital where the male should, according to the theoretical principle, have the same height or be higher than the female. These capital types should, from the perspective of capital types, be approached holistically because they can be exchanged internally [29]. For example, short men such as Martin Scorsese $(163 \mathrm{~cm})$ or Vladimir Putin $(170 \mathrm{~cm})$ offer other types of capital and status, which outperforms height, according to Bourdieu's reasoning [28]. Similarly, a male's low educational level can be outperformed by his high income or high occupational rank (managerial level). In terms of homogamy-dimensions, however, a match should share culture and religion. Also shared proximity is found to be important when people choose a partner: a potential match should preferably live geographically close to one another.

However, while social science stresses the importance of socioeconomic principles for the creation of life-long partnerships, where background, social class, and the wider context of relationships are placed in the foreground, the psychological mating literature stresses individual and personal aspects as essential for mating. Such psychological aspects include personality, a person's need for intimacy, tidiness, and communication preferences as important when choosing a partner [30]. However, although psychologicalaspects are of importance when choosing a partner, these aspects play a larger role when being in a relationship, rather than prior to establishing a relationship [30]. As matching algorithms seek to establish a connection between two persons that do not know each other, they should - if following the logic of Bourdieu's social theory of capital types [28] - take socioeconomic aspects into close consideration when calculating and recommending matches.

By having insights into socioeconomic mating principles or “domain knowledge," using the reversed engineering method, offline mating practices and the choice of partner with the model for how dating platforms calculate matches was tested and compared. By doing so, it was possible to see what dating platforms prioritize when suggesting that two persons are a good match.

\section{Methodology}

\subsection{Experiment}

To empirically understand how the algorithms used by dating platforms calculate suitable matches and how the system defines what makes a "good" match, the method of reversed engineering was chosen as the most suitable research design for the experiment. An experiment is a research design method that uses manipulation and controlled testing to understand causal relationships [31]. An experiment manipulates one or several variables to observe the effect of other dependent variables. However, despite designing the study as an experiment, an inductive approach incorporating qualitative methods is used to analyze and interpret the collected data. Thus, this study is positioned within an interpretative paradigm, understanding that reality is created by individuals and that reality therefore needs to be interpreted in depth and through a qualitative lens.

The experiment began with a close analysis of the Norwegian dating platform market. It was then decided on a sample consisting of four well-established and recognized online dating platforms that market themselves as dating sites for heterosexual single persons seeking long-lasting and serious relationships. The platforms in our sample were Match, The Meeting Place (Møteplassen), Sugar (Sukker), and Academic Singles.

\subsection{Pilot study}

To gather key insights for designing the experiment, a pilot study was done in January and 
February 2019 before conducting the experiment in April and May 2019. The results of the pilot study highlighted the importance of creating all six ghost profiles (GPs) (see below) on the same platform within a short time (within a few hours on the same day) to make sure that the match suggestions from the platform were based on the exact same sample of profiles present in the platform. Other key insights learned from the pilot study included the importance of designing GPs that were strong representations of individuals seeking long-lasting relationships and being in a lifephase where one typically wants to settle down and grow a family. During the pilot, the full process for becoming a member of the dating site was collected (as screenshots) for all four dating platforms. This was important so I could accurately plan in detail what the GPs should fill in when creating a profile in the experiment. It was essential that the six GPs (see below) answered the platform's questionnaires, so that the platform's matching suggestions could not be explained due to different answers in the survey.

\subsection{Ghost profiles}

Four GPs (fake accounts) was created: two males (M1 and M2) and two females (F1 and F2) on the four dating platforms. Two control GPs (F3 and M3) was in addition created on the four platforms to control for the hypergamy aspects (height, educational level, occupation, and income) and homogamy dimensions of religion and proximity that we wanted to test. Thus, six GPs was created as test persons. The GPs had the following characteristics:

Male 1 (M1):

- 32 years (age)

- $\quad 1.69 \mathrm{~cm}$ (height)

- High school(educational level)

- Truck driver (occupation)

- 400,000 $\mathrm{NOK}^{1}$ (income)

- Christian (religion)

- $\quad$ Oslo, Norway (proximity)

Male 2 (M2)

- 32 years (age)

- $1.69 \mathrm{~cm}$ (height)

- $\quad$ Ph.D. (educational level)

- Medical doctor (occupation)

- $1,000,000$ NOK (income)

- $\quad$ Christian (religion)

- $\quad$ Oslo, Norway (proximity)

Male 3 (M3)—control person

1 100, 000 NOK $=10,800$ USD



Female 1 (F1)

- 32 years (age)

- $\quad 1.76 \mathrm{~cm}$ (height)

- High school(educational level)

- Hairdresser (occupation)

- 400,000 NOK (income)

- Christian (religion)

- $\quad$ Oslo, Norway (proximity)

Female 2 (F2)

- 32 years (age)

- $\quad 1.76 \mathrm{~cm}$ (height)

- $\quad$ Ph.D. (educational level)

- Medical doctor (occupation)

- $1,000,000 \mathrm{NOK}$ (income)

- Christian (religion)

- $\quad$ Oslo, Norway (proximity)

Female 3 (F3)_control person

- 32 years (age)

- $\quad 1.67 \mathrm{~cm}$ (height)

- Master (educational level)

- $\quad$ Consultant (occupation)

- 600,000 NOK (income)

- $\quad$ Christian (religion)

- Oslo, Norway (proximity)

As the GPs characteristics show, M1 and M2 have the same age, height, religion, and proximity, but different educational levels (low versus high), incomes (low versus high), and occupations (working class (i.e., truck driver, hairdresser) versus higher-middle class (i.e., medical doctor)). The two female GPs have a similar logic. To control for the socioeconomic principles, two control GPs was created-one male and one female (M3 \& F3). These control persons are more average representations than the other GPs in terms of height (1.80 cm for men, $1.67 \mathrm{~cm}$ for woman), income 600,000 NOK (the average income in Norway in 2018 was 547,320 NOK), educational level (master's degree), and occupation (consultant). All six GPs were Christian (religion) and lived in Oslo, the capital of Norway (suggesting a robust pool of candidates from which the platform could choose matches). Except for these socioeconomic differences, the six GPs answered all questions in the initial questionnaire identically. The 
GPs provided quite average answers about the degree of tidiness, hobbies, and food and drinking preferences.

Because of research ethics, none of the GPs uploaded a profile picture, and any required profile text was kept as anonymous as possible. None of the GPs replied to messages sent to them, and the profiles were deleted shortly after the data were collected.

\subsection{Data analysis}

Input data from the process to become a member was gathered as screenshots during the pilot study, including all the questions asked by the platforms and the answer categories. An analysis of the questions posed by the platforms and the response alternatives offered was also performed. I examined whether and what way socioeconomic aspects and the hypergamy principle was covered or embedded in the questions asked by the four platforms. The questions from all the platforms were then classified into categories based on their characteristics (e.g., questions about personality, expectations from a partner, communication needs, and hobbies) for an in-depth analysis. I collected Output data for the top five matches the platforms suggested for all six GPs was collected, including the match-score and rank and the hypergamy (income, educational level, occupational level, height) and homogamy (religion, proximity) dimensions. This data was coded into tables and analyzed internally and horizontally. The four platforms were also examined in-depth to see how they market their platforms, identify interaction opportunities to search for platform members, and record any differences for paying versus non-paying members.

\section{Reversed engineering step by step}

As stated in section 2.1., an input-throughputoutput model was used to examine the functioning of algorithmic selection used by dating platforms. The data collection and analysis process followed three steps: (1) Creating a profile (input), (2) the matching process (throughput), and (3) the recommended matches (output). Section 4.1 - 4.3 describes these steps.

\subsection{Step one: Creating a profile (input)}

As a starting point, all the six GPs was created in each platform. To become a platform member, the user has to answer a number of questions. Because the pilot study revealed which questions each platform would ask, the same answers for all six profiles was given. Different answers were provided to the different socioeconomic variables for the six GPs in order to test whether the platform took these into considerations when calculating the recommended matches.

This first step provided the platform with input data. The four platforms differed in the number of questions asked in this phase, varying from 33 questions (Academic Singles) to almost 200 (The Meeting Place).

The questions posed to applicants by all four platforms could be grouped into seven categories: (1) expectations about relationships $(\mathrm{N}=70)$, (2) desired characteristics of a partner $(\mathrm{N}=40)$, (3) the applicant's personality $(\mathrm{N}=178)$, (4) the applicant's values/attitudes ( $\mathrm{N}=43)$, (5) the applicant's taste, hobbies, and interests $(\mathrm{N}=28)$, (6) communication needs $(\mathrm{N}=9)$, and $(7)$ personalia and practical information (e.g., inclusive thirst for knowledge, IQ; N $=63$ ).

As can be seen, the majority of questions concerned the applicant's personality, followed by expectations of being in a relationship, and personalia and practical information. Although aspects related to socioeconomic and hypergamy dimensions were embedded to some extent in the latter category, it was found that the platforms barely use the input in this category when matching candidates (see the output section).

Thus, the platforms focused mainly on the applicant's personality and other personal characteristics clearly influenced by psychological perspectives that stress the individual's characteristics and needs. For example, the majority of the questions asked in the platforms concern psychological factors. On The Meeting Place platform, 104 of 190 questions had a clear reference to questions typical of psychological and personality tests, such as The Big Five test and Myers-Briggs Type Indicator personality test.

Moreover, the input for all four platforms had severe methodological weaknesses in terms of their inadequate response options, especially when it came to important socioeconomic and hypergamy dimensions. For example, the variable “income” was measured by three of the four platforms as a categorical variable with very inadequate reply categories, while the fourth platform (Sugar) measured "income" as a numeric variable (from 1 to 10) (Table 1). Note that the average income in Norway (which was 547,320 NOK in 2018) falls into the boxes marked in grey, which are closer to the right side of the continuum shown in Table 1. Following a survey methodology and logic, the category for 'average income' should be more centered to the middle of the scale, rather than close to the end (right side) of the scale. As the responsecategories stand now, they are very fine-tuned up to 
average income, and less fortunate beyond average. This leaves the system with less accurate responsecategories to calculate on. Moreover, many of the response categories concerning socio-economic aspects are not mutually exclusive (e.g. '100 000 - 200 000' and '200 $000-300000$ ’).

Table 1. Response categories for income in the four dating platforms

\begin{tabular}{|c|c|c|c|c|c|c|c|c|}
\hline \multirow{2}{*}{$\begin{array}{l}\begin{array}{l}\text { Academic } \\
\text { singles }\end{array} \\
\text { Match }\end{array}$} & \multicolumn{2}{|c|}{$150000-250000$} & \multicolumn{2}{|c|}{$250000-400000$} & \multirow{2}{*}{$\begin{array}{l}400000 \\
-550000 \\
300000- \\
400000\end{array}$} & \multirow{2}{*}{$\begin{array}{l}550000 \\
-700000 \\
400000- \\
500000\end{array}$} & \multicolumn{2}{|c|}{ More than 700000} \\
\hline & $\begin{array}{l}\text { Don't } \\
\text { want to } \\
\text { answer }\end{array}$ & $\begin{array}{l}\text { Less } \\
\text { than } \\
100000\end{array}$ & $\begin{array}{l}100000- \\
200000\end{array}$ & $\begin{array}{l}200000- \\
300000\end{array}$ & & & $\begin{array}{l}500000- \\
1000 \\
000\end{array}$ & $\begin{array}{l}\text { More } \\
\text { than } \\
1000000\end{array}$ \\
\hline $\begin{array}{l}\text { The } \\
\text { meeting } \\
\text { place }\end{array}$ & $\begin{array}{l}\text { Don't } \\
\text { want to } \\
\text { state }\end{array}$ & $\begin{array}{l}\text { Less } \\
\text { than } \\
100000\end{array}$ & $\begin{array}{l}100000- \\
200000\end{array}$ & $\begin{array}{l}201000- \\
300000\end{array}$ & $\begin{array}{l}301000- \\
399000\end{array}$ & $\begin{array}{l}400000- \\
499000\end{array}$ & $\begin{array}{l}500000- \\
599000\end{array}$ & $\begin{array}{l}\text { More } \\
\text { than } \\
600000\end{array}$ \\
\hline Sugar & \multicolumn{3}{|c|}{$\begin{array}{l}\text { Borrows money and constantly lags } \\
\text { behind - value } 1\end{array}$} & \multicolumn{4}{|c|}{$2-9$} & $\begin{array}{l}\text { Have } \\
\text { plenty of } \\
\text { money - } \\
\text { value } 10\end{array}$ \\
\hline
\end{tabular}

Thus, the input data concerning the socioeconomic aspects (i.e., income, educational level, and occupation) used by the dating platforms in their matching all suffer from severe methodological weaknesses and flaws (e.g. unfortunate non-exclusive response categories). As we will see later in section 4.3., these flaws influence the platforms' outputs and recommendations that are presented to users as good matches.

This first step in the reversed engineering process collects data on the kind of input that the user provides to the system based on the response alternatives to the questions offered by the platform.

\subsection{Step two: The matching process (throughput)}

The throughput process is characterized by the platform or media assigning relevance and making selections based on the input data (see Figure 1). Here, different codes and operating modes (e.g., matching or sorting algorithms) knead the input data obtained from the user in ways that is decided by the model the platform is programmed to follow. Since dating platforms follow a content-based model in their recommendation systems, the platforms base their recommendations on additional information obtained from the users [2].

On dating platforms, the content the user provides as input to the system is weighted in the throughput phase. We can observe how the algorithms in this throughput phase work by examining how the system represents and presents the user's personal profile, which forms the basis of how the system calculates its matches. All four platforms state that they use different scientific tests as a backdrop for how their system weights relevance. For example, Match claims to use an "attraction test" that is specially designed by their AssesFirst-team, which comprises "an expert group consisting of psychologists and psychometric tests” [32]. After the user has set up a profile, the platform presents the user's profile to the opposite sex from a psychological perspective based on the user's scores for different psychological attributes. As an example, the Academic Singles platform calculates a personality report based on the user's input. The platform claims that the report "helps us determine your personal profile (...) to identify personal characteristics (...), define your behavior in a relationship and help you understand the seven dimensions in a harmonic relationship" [33]. In the throughput phase, the algorithms of the dating platforms calculate matches from the perspective of psychological mating preferences based on similarities in the users' inputs; that is, the more similar the two persons answered the questions asked in the input phase, the closer the match. The extent to which two users are similar and share psychological characteristics thus determines the platform's recommendations in a ranked list and influences how the platform matches people.

The second step in the reversed engineering process involved analyzing how the platforms present the personal profiles and how they represent the user after they become a platform member. The personal profile is characterized by a psychological approach (i.e., the person's needs, preferences, and personality).

\subsection{Step three: Recommended matches (output)}

The outputs of the platforms assume different forms or orders (e.g., rankings and recommendations). Most of the dating platforms recommend matches in a ranked order, where "the best match" is typically ranked as number one and/or placed at the top of a list of matches, sorted based on the degree of a matching score between two users. However, this is not always the case, as some platforms give rank benefits to paying users. Some platforms also provide new members with better rank - or visibility - than those who have been members of the platform for a while. However, this assumption is mainly speculation since it was difficult to test this observation thoroughly.

A "match" denotes what the platform decides is a good fit between two heterosexual single persons looking for a long-term and serious relationship. collected Output data concerning the hypergamy dimensions (i.e., income, educational level, occupation, height) and key homogamy aspects (i.e., religion, proximity) was collected from the top five matches the platforms suggested for the six GPs on the four platforms. Details about the match-scores and ranks of these matches was collected and analyzed how the platforms articulated why they were a good match. 
This data was coded into tables and analyzed the details internally and horizontally.

If the platforms took socioeconomic and the hypergamy aspects into account in their matching and recommendations, the platform should provide the female user with candidates that have several of the following attributes:

- a similar or higher educational level

- a similar or higher income

- a similar or higher occupational level or status

- a similar height or taller

- similar religious beliefs

Similarly, following the same theoretical logic, the male profiles would be presented with a list of female candidates that hold several of the following attributes:

- a similar or lower educational level

- a similar or lower income

- a similar or lower occupational level or status

- a similar height or shorter

- similar religious beliefs

As stated earlier in section 2.2., these items or variables can, according to Bourdieu [28], be exchanged internally. For example, educational level can be outperformed by a high income from a male perspective. However, an analysis of the output data shows that none of the algorithms of the four platforms take these aspects into account when performing their matching. While two of the platforms (Academic Singles and Sugar) took height into account, they ignored the logic of exchangeability. Nevertheless, all the GPs were matched with profiles based on the degree of similarity between the two persons' input data concerning psychological and personal issues. This was exemplified by (1) all the GPs being matched with the same pool of candidates and (2) the female GPs being matched with the male GPs. Thus, several of the platforms suggested the truck driver with low income and low educational level (M1) and the medical doctor with a high income and a Ph.D. (F2) as their best match. As mentioned above, two of the platforms (Academic Singles and Sugar) took height into account and thus excluded two male GPs from the female candidates' pool of matches that were taller than them. M2, who had a high income, educational level, and occupation (medical doctor), was also excluded, despite having socioeconomic aspects that outperform height. The match score given by the platform thus represents the extent to which two persons replied similarly to questions based on psychological aspects. The throughput phase, which weights the relevance and selects what the platform should prioritize in their matching, is therefore based on the degree of similarities among answers given to a specific group of answers. This category of questions and input concerns psychological aspects, such as personality, expectations from a relationship, degree of tidiness, temper, and communication needs. This finding shows that (1) the majority of the questions asked by the platform (input) concerns the user's personality $(\mathrm{N}=178)$, followed by their expectations of being in a relationship ( $\mathrm{N}=70)$, and (2) the third biggest category of questions "personalia and practical information” $(\mathrm{N}=63)$, which covers some socioeconomic and hypergamy aspects, is not taken into account in the matching process. A key finding from the input-section, was that all four platforms suffered from severe methodological weaknesses in terms of their inadequate variables and response options (e.g. overlapping and/or inadequate response categories). Poor quality in the input logically travel into the output, in which also could shed light into why the platform ignores key socio-economic aspects. On the other hand, poor response categories could might as well illustrate that the platform do not take socioeconomic aspects seriously into consideration when suggesting matches. This is supported by the fact that none of the four platforms took religion or proximity into account when recommending matches for longlasting close relationships.

These findings suggest that the theoretical model used by dating platforms, which use content-based methods to make recommendations to users, is influenced by a psychological discourse and similarities in the content provided to the system by the users. In content-based methods, new users or items are described by their characteristics (content) and relevant suggestions can thus be made for these new entities. Input in dating platforms is used to predict whether a user fits with—or "likes" —an item.

The theoretical model behind the recommendation system of all four platforms is also used in their marketing to attract customers. For example, Academic Singles states that their "advanced matching systemis a pure success formula," stressing that their system only matches a person with candidates with whom they have things in common and that some advantages of their platform are the use of scientific personality tests and suggestions of partners, which give a perfect fit with their personality [33].

The third step in the reversed engineering process involves examining the characteristics of the candidates that the platforms recommend as strong candidates for the user. This step also involves examining how the platforms rank the potential candidates and studying candidates that are not suggested as good matches with the applicant. Finally, a close analysis of the platforms' affordances was 
performed (e.g., the user's opportunities to independently search for candidates, the ability to contact others, and the different abilities of paying versus non-paying members to explore others' profiles).

\section{Discussion and concluding remarks}

Our daily activities and our media consumption are increasingly being shaped by algorithms operating behind the scenes. More insights into how algorithms empirically work is critical given their increasing importance in shaping social and economic life $[6,10]$. By using a reversed engineering method and the simple input-throughput-output model offered by Just and Latzer [13], this study examined how algorithms used by four dating platforms calculate and rank single heterosexual persons seeking a partner for life. By setting up a carefully planned experiment, the study at hand examined the functioning of algorithmic selection on dating platforms using a basic input-throughputoutput model that reversed the engineering process. This method enabled the exploration of the algorithms of dating platforms, perhaps more from the outside and in, than from the inside-out $[6,10]$. In this way, it was possible to pay attention to what algorithms do (match people based on similarities and along psychological axes, leaving socioeconomic principles out from their theoretical matching model) by making the "black box" of the matching machinery available through leaks, openings, and shortcomings that we could observe. By directing the attention to the systems' functions, by testing the inputs and outputs of these systems, and by studying the outcomes of the users' choices and interactions with the dating platform, a better understanding of the mechanisms employed by dating platform algorithms was obtained.

This study supports the notion that algorithms do not operate in a technical vacuum and cannot be divorced from the underlying perspectives the system is modelled upon because the code, algorithms, and computer systems contain the embedded values held by the individuals who construct them [23-25]. In this study, the algorithms were found to be influenced by a psychological discourse and values. The platforms' input, throughput, and output stages weighted relevance to psychological aspects. However, the social science literature on homogamy and hypergamy, find that socioeconomic aspects are important for heterosexual peoples' choice of long-term relationships [27]. If socioeconomic resources or capital (e.g., social class, rank, background, income, occupational prestige, amongst others) are essential when choosing a partner, and because capital is convertible (it can be exchanged into different types of capital) [28], the matching process of dating platforms should in the future take socioeconomic aspects into holistic consideration in their calculations when matching single persons for long-lasting relationships.

From an algorithmic culture angle, this study illustrated that the pool of candidates the four dating platforms recommend as matches are based on selfreported similarities between the matches. Thus, the user is presented and surrounded with a pool of partners similar to the user, where dissimilar candidates are ignored and made less relevant, and thus less accessible. This reminds of a 'more of the same' logic that characterizes recommendations used by Amazon, Netflix, Spotify and other platforms. In its nature, recommendation systems seek to overcome the information overload problem and suggest relevant items or content, such as movies to watch, text to read, products to buy - or candidates to partner with. However, a 'more of the same' model and logic in the context of dating platforms not only ignore socioeconomic elements that the social science finds important when choosing lifelong partnerships, it also imposes a 'threat of invisibility', in many ways similar to Bucher's findings of Facebook's algorithms [8]. The algorithmic machinery of dating platforms control to a large extent what pool of candidates the user gain access to. Accessibility and availability to the pool of single candidates is, however, at the same time closely related to the strategic commercial interests of the platforms. Paying members, for example, gain more or wider access to the pool of singles, as well as other benefits such as better visibility than non-paying members.

This experiment was very time-consuming. During the data collection process the GPs were kicked out of the platform several times and the full experiment had to be repeated since it was important to collect data from the GPs within the same platform on the exact same day to ensure that the platform's sample contained the same pool of candidates.

This paper presented an example of how to empirically approach and evaluate the digital data collection process and how to interpret findings from this type of research. Hopefully, this study inspires other media and information system researchers to use a reversed engineering method in a time where media actors and platform owners are reluctant to share how their algorithms work. Additionally, much of the existing methodological literature is typically tool or technology driven rather than a result of empirical examination of the data collection process. 


\section{Acknowledgements}

Many thanks to professor Runar Døving from Kristiania University College in Norway, whom I collaborated with in this study of dating platforms.

\section{References}

[1] Latzer M, Hollnbuchner K, Just, Saurwein F. The Economics of Algorithmic Selection on the Internet, Working Paper-Media Change \& Innovation Division, 2014.

[2] Rocca B. Introduction to Recommender Systems. Overview of some Major Recommendation Algorithms, Towards Datascience, 2019. Extracted June 3, 2020.

[3] Seaver N. Knowing algorithms. 2014.

[4]. Carah N, Angus D, Thomas D. media|machines [Internet]. Carah N, editor. http://mediamachines.org/: Nicholas Carah. 2017. [cited 2020]. Available from: http://mediamachines.org/log/2017/8/15/algorithmic-cultureand-machine-learning.

[5]. Hallinan B, Striphas T. Recommended for you: The Netflix Prize and the production of algorithmic culture. New media \& society. 2016;18(1):117-37.

[6]. Kitchin R. Thinking critically about and researching algorithms. Information, Communication \& Society. 2017;20(1):14-29.

[7]. Gillespie T, Boczkowski PJ, Foot KA. Media technologies: Essays on communication, materiality, and society. Massachusetts, USA: MIT Press; 2014. [8]. Bucher T. Want to be on the top? Algorithmic power and the threat of invisibility on Facebook. New Media \& Society. 2012;14(7):1164-80.

[9]. Carr A. I Found Out My Secret Internal Tinder Rating And Now I Wish I Hadn't https:/www.fastcompany.com/: Fast Company; 2016 [Available from: https://www.fastcomp any.com/3054871/whats-y our-tinderscore-inside-the-apps-internal-ranking-system.

[10]. Bucher T. If... Then: Algorithmic power and politics: Oxford University Press; 2018.

[11]. Finn E. What algorithms want: Imagination in the age of computing: MIT Press; 2017.

[12]. Snickars P. More of the Same-On Spotify Radio. Culture Unbound Journal of Current Cultural Research. 2017;9(2):184-211.

[13]. Just N, Latzer M. Governance by algorithms: reality construction by algorithmic selection on the Internet. Media, Culture \& Society. 2017;39(2):238-58.

[14]. Rosenfeld MJ, Thomas RJ, Hausen S. Disintermediating your friends: How online dating in the United States displaces other ways of meeting. Proceedings of the National Academy of Sciences. 2019;116(36).
[15]. Brooks A. A Timeline of How Online Dating Has Changed Over the Last 20+ Years. https://wwwdatingnewscom/. 2018 July 13.

[16]. Barraket J, Henry-Waring MS. Getting it on (line) Sociological perspectives on e-dating. Journal of Sociology. 2008;44(2):149-65.

[17]. Henry-Waring M, Barraket J. Dating \& intimacy in the 21 st century: The use of online dating sites in Australia. International Journal of Emerging Technologies \& Society. 2008;6(1).

[18]. Bauman Z. Intimations of postmodernity: Routledge; 2003.

[19]. Resnick P, Varian HR. Recommender systems. Communications of the ACM.1997;40(3):56-8.

[20]. Ricci F, Rokach L, Shapira B. Introduction to recommender systems handbook. Recommender systems handbook: Springer; 2011. p. 1-35.

[21]. Krumpal I. Determinants of social desirability bias in sensitive surveys: a literature review. Quality \& Quantity. 2013;47(4):2025-47.

[22]. Joel S, Eastwick PW, Finkel EJ. Is romantic desire predictable? Machine learning applied to initial romantic attraction. Psychological science. 2017;28(10):1478-89.

[23]. Elish MC, boyd d. Situating methods in the magic of Big Data and AI. Communication Monographs. 2018;85(1):57-80.

[24]. Forsythe D. Studying those who study us: An anthropologist in the world of artificial intelligence: Stanford University Press; 2001.

[25]. Geiger RS. Bots, bespoke, code and the materiality of software platforms. Information, Communication \& Society. 2014;17(3):342-56.

[26]. Diakopoulos N. Algorithmic accountability reporting: On the investigation of black boxes [A Tow/Knight Brief]. Tow Center for Digital Journalism, Columbia Journalism School, New York, NY Retrieved from http://towcenter org/algorithmic-accountability-2. 2013.

[27]. Mohanadoss T. Hypergamy and its inherent contradictions. Anthropos. 1995(H. 4./6):558-63. [28]. Bourdieu P. The forms of capital. In: Richardson JG, editor. Handbook of theory and research for the sociology of education New York: Greenwood Press; 1986. p. 241-58.

[29]. Chudnovskaya M. Housing context and childbearing in Sweden: a cohort study. Housing Studies. 2019;34(3):469-88.

[30]. Pettersen L, Døving R. It's a mismatch! - A study of dating platforms' recommendation systems. Forthcoming. [31]. Shadish WR, Cook TD, Campbell D. Experimental and quasi-experimental designs for generalized causal inference: Boston: Houghton Mifflin Company; 2002.

[32]. Please refer to Match https://no.match.com

[33]. Please refer to Academic singles www.academicsingles.no 